\title{
ON THE CONSTRUCTIBILITY OF PRIME CHARACTERISTIC PERIODIC ASSOCIATIVE AND JORDAN RINGS
}

\author{
BY
}

\section{J. A. LOUSTAU}

ABSTRACT. The object of this paper is to show that any periodic associative ring of prime characteristic can be embedded in a periodic associative ring of prime characteristic which is constructible from a relatively complemented, distributive lattic and a family of periodic fields. Further, it will be proved that any periodic Jordan ring of prime characteristic is also embeddable in a periodic Jordan ring which is constructible from a lattice of the above type and a family of periodic Jordan rings of a symmetric bilinear form.

1. Introduction. As in [1] a power-associative ring $R$ is called periodic if for every element $x$ of $R$ there exists an integer $n(x)>1$, depending on $x$, with $x=x^{n(x)}$. Further, as in [1] it is known that any power-associative periodic ring is a discrete direct sum of prime characteristic periodic rings. Moreover, Osborn gave in [1] a procedure for constructing a prime characteristic, associative, periodic ring from a relatively complemented, distributive lattice and a family of periodic fields, and he asked if every such ring was constructible.

This paper will be concerned with the constructibility of prime characteristic periodic associative and Jordan rings. We will first show that in the associative case, any periodic, prime characteristic ring is embeddable in a constructible periodic ring. For Jordan periodic rings, we know from [2] that the characteristic 2 case is associative, and hence we only need consider Jordan rings of characteristic $p$ different from 2 . If $J$ is such a Jordan periodic ring, then we shall refine the structure theorem given for $J$ in [1] using techniques from sheaf theory. Then with this refinement we will be able to embed $J$ in a constructible prime characteristic Jordan periodic ring, $\bar{J}$. Now $\bar{J}$ will be constructible from a relatively complemented distributive lattice and a family of periodic Jordan rings of a symmetric bilinear form; further the constructive procedure to be given has the property that it reduces to the one given in [1] if the family of Jordan rings is a family of periodic fields. Finally, we shall show by example that not every prime characteristic Jordan periodic ring is constructible in this manner. Our first task is to present the constructive procedure. 
Let $E$ be a distributive lattice which is relatively complemented in the sense that if $e_{\alpha}, e_{\beta}, e_{\gamma} \in E$ with $e_{\alpha} \leqslant e_{\beta} \leqslant e_{\gamma}$, then there exists $e_{\beta}^{\prime} \in E$ with $e_{\beta} \wedge e_{\beta}^{\prime}=e_{\alpha}$ and $e_{\beta} \vee e_{\beta}^{\prime}=e_{\gamma}$. Now let $\left\{J_{\alpha}: e_{\alpha} \in E, e_{\alpha} \neq 0\right\}$ be a family of Jordan periodic rings of a symmetric bilinear form and of characteristic $p \neq 2$. Then each $J_{\alpha}$ is a periodic field, the direct sum of two periodic fields, or there exist a periodic field $\Phi_{\alpha}$ and $u \in \Phi_{\alpha}$ with

$$
J_{\alpha}=\left\{\left(\begin{array}{cc}
a & b u \\
b & c
\end{array}\right): a, b, c \in \Phi_{\alpha} \text { and }-u \text { is a nonsquare }\right\}
$$

with respect to the Jordan product on the $2 \times 2$ matrix ring over $\Phi_{\alpha}$ [1]. Hence if $\Omega$ is the algebraic closure of $Z_{p}$, the integers modulo $p$, then in a natural way each $J_{\alpha}$ can be imbedded in $\Omega_{2}^{+}$, the $2 \times 2$ matrix ring over $\Omega$ with the Jordan product. In particular, the image of the identity of $J_{\alpha}$ in $\Omega_{2}$ is the identity. Now we shall require that the family $\left\{J_{\alpha}: e_{\alpha} \in E, e_{\alpha} \neq 0\right\}$ has the property that $J_{\alpha} \subseteq J_{\beta}$ whenever $e_{\alpha} \geqslant e_{\beta} \neq 0$ where each $J_{\alpha}$ is considered to be contained in $\Omega_{2}^{+}$. As in [1], if $0 \neq e_{\beta}$ and $0 \neq e_{\gamma}$ are in $E$ with $e_{\beta} \wedge e_{\gamma}=0$ and $e_{\beta} \vee e_{\gamma}=e_{\alpha}$ then $J_{\alpha}=J_{\beta} \cap J_{\gamma}$. With this lattice $E$ and set $\left\{J_{\alpha}: e_{\alpha} \in E, e_{\alpha} \neq 0\right\}$ we can construct a prime characteristic Jordan periodic ring.

Let $L$ be the free abelian group generated by the pairs $\left(c_{\alpha}, e_{\alpha}\right)$ where $e_{\alpha}$ is a nonzero element of $E$ and $c_{\alpha} \in J_{\alpha}$, and define

$$
\left(c_{\alpha}, e_{\alpha}\right)\left(c_{\beta}, e_{\beta}\right)= \begin{cases}\left(c_{\alpha} c_{\beta}, e_{\alpha} \wedge e_{\beta}\right) & \text { if } e_{\alpha} \wedge e_{\beta} \neq 0 \\ 0 & \text { if } e_{\alpha} \wedge e_{\beta}=0\end{cases}
$$

where the product $c_{\alpha} c_{\beta}$ is in $J_{\alpha} \cap J_{\beta} \subset \Omega_{2}^{+}$. It is immediate that with (1), $L$ forms a Jordan ring. Next let $I$ be the ideal of $L$ generated by all elements of $L$ of the form

$$
\left(c_{\alpha}+c_{\alpha}^{\prime}, e_{\alpha}\right)-\left(c_{\alpha}, e_{\alpha}\right)-\left(c_{\alpha}^{\prime}, e_{\alpha}\right)
$$

for $c_{\alpha}, c_{\alpha}^{\prime} \in J_{\alpha}$ and $e_{\alpha} \in E$, or of the form

$$
\left(c_{\gamma}, e_{\alpha} \vee e_{\beta}\right)-\left(c_{\gamma}, e_{\alpha}\right)-\left(c_{\gamma}, e_{\beta}\right),
$$

for $c_{\gamma} \in J_{\alpha} \cap J_{\beta}, e_{\alpha}, e_{\beta} \in E$, and $e_{\alpha} \wedge e_{\beta}=0$. We claim that $L / I$ is a periodic Jordan ring of characteristic $p$. 
Let $c_{\alpha} * e_{\alpha}$ denote the image of $\left(c_{\alpha}, e_{\alpha}\right)$ in $L / I$, then equations (1), (2), and (3) imply

$$
\begin{aligned}
\left(c_{\alpha} * e_{\alpha}\right)\left(c_{\beta} * e_{\beta}\right) & =c_{\alpha} c_{\beta} *\left(e_{\alpha} \wedge e_{\beta}\right), \\
\left(c_{\alpha}+c_{\alpha}^{\prime}\right) * e_{\alpha} & =\left(c_{\alpha} * e_{\alpha}\right)+\left(c_{\alpha}^{\prime} * e_{\alpha}\right), \\
c_{\gamma} *\left(e_{\alpha} \vee e_{\beta}\right) & =c_{\gamma} * e_{\alpha}+c_{\gamma} * e_{\beta}
\end{aligned}
$$

where in (6), $e_{\alpha} \wedge e_{\beta}=0$. Now by (5), $p\left(c_{\alpha} * e_{\alpha}\right)=p c_{\alpha} * e_{\alpha}=0$, so that $L / I$ has characteristic $p$. Moreover, as in [1] equations (4) and (6) imply that for each $c \in L / I$, there exist mutually orthogonal, nonzero elements $e_{\alpha}, e_{\beta}, \cdots$, $e_{\rho} \in E$ and elements $c_{\alpha} \in J_{\alpha}, c_{\beta} \in J_{\beta}, \cdots, c_{\rho} \in J_{\rho}$, with

$$
c=c_{\alpha} * e_{\alpha}+c_{\beta} * e_{\beta}+\cdots+c_{\rho} * e_{\rho} .
$$

Then if $m$ is taken so that $c_{\alpha}^{m}=c_{\alpha}, \cdots, c_{\rho}^{m}=c_{\rho}$, it follows from (4), that $c^{m}=c$ and $L / I$ is periodic, and hence $E$ and $\left\{J_{\alpha}: e_{\alpha} \in E, e_{\alpha} \neq 0\right\}$ gives rise to a prime characteristic, periodic, Jordan ring as claimed. Furthermore, suppose that if $J_{\alpha}$ is not simple, then there exists $e_{\beta}<e_{\alpha}$ with $J_{\beta}$ simple. With this assumption $\left\{1 * e_{\alpha}: e_{\alpha} \in E\right\}$ is the set of central idempotents of $L / I$ and thus $E$ is isomorphic to the lattice of central idempotents of $L / I$. Finally, if each $J_{\alpha}$ is a periodic field, then $L / I$ is an associative periodic ring.

2. The associative case. Let $R$ be an associative periodic ring of characteristic.$p$, a prime integer. Hence, $R$ is isomorphic to a subdirect sum of periodic fields $F_{i}$, where $i$ is an element of some index set $\Lambda$. If we let $\Omega$, as above, denote the algebraic closure of $Z_{p}$, then each $F_{i}$ can be considered to be a subfield of $\Omega$. Now let $S$ be the complete direct sum of the fields $\Omega_{i}, i \in \Lambda$, where each $\Omega_{i}$ is a copy of $\Omega$. Further, for each $x$ in $S$, let $x_{i}$ denote the image of $x$ under the projection of $S$ onto $\Omega_{i}$.

Define $\bar{R}=\left\{x \in S\right.$ : only a finite number of the $x_{i}$ are distinct $\}$. It is immediate that $\bar{R}$ is a periodic ring of characteristic $p$. If $\bar{E}$ is the lattice of idempotents of $\bar{R}$, then $\bar{E}$ is isomorphic to the power set $P(\Lambda)$ of $\Lambda$ under the mapping $\phi: \bar{E} \rightarrow P(\Lambda)$ given by $\phi(e)=A \subseteq \Lambda$ if $A=\left\{i \in \Lambda: e_{i} \neq 0\right\}$. Also for every $e \in \bar{E}$, the maximal subfields of $\left\{x \in \bar{R}: e=x^{n(x)-1}\right\}$ are just $\Omega$. Thus, in the terminology of [1], $\bar{R}$ is complete, and hence, is constructible .

We claim now that $R \subseteq \bar{R}$. It is clear that $R \subseteq S$. However, if $x \in R$ and $n>1$ with $x^{n}=x$, then $x_{i}^{n}=x_{i}$ for every $i \in \Lambda$. Let $K \subseteq \Omega$ be 
the splitting field of $X^{n}-X$ over $Z_{p}$; then $K$ is finite and for each $i, x_{i} \in K$. But then $x \in \bar{R}$, so indeed $R \subseteq \bar{R}$. We have proved the following theorem.

THEOREM 1. Let $R$ be an associative periodic ring of prime characteristic $p$, then $R$ is imbeddable in a constructible prime characteristic periodic ring.

3. A structure theorem. Before proving the second main result of this paper, it is necessary to strengthen the structure theorem given in [1] for Jordan periodic rings. In particular, in [1] and [2] it is proved that any Jordan periodic ring is isomorphic to a subdirect sum of Jordan rings $\left\{J_{i}: i \in \Lambda\right\}$ where each $J_{i}$ is either a periodic field or else there exists a periodic field $\Phi_{i}$ and $u \in \Phi_{i}$ such that

$$
J=\left\{\left(\begin{array}{cc}
a & b u \\
b & c
\end{array}\right): a, b, c \in \Phi_{i} \text { and }-u \text { is a nonsquare in } \Phi_{i}\right\}
$$

with respect to the Jordan product. Thus, if $J$ is a Jordan periodic ring of characteristic $p$ different from 2 , then $J$ has the above structure with the additional property that each $J_{i}$ is contained in $\Omega_{2}^{+}$, where $\Omega$ is the algebraic closure of $Z_{p}$. Now if for each $i, \phi_{i}: J \rightarrow J_{i}$ denotes the $i$ th projection of $J$, then it is our aim to find a representation of $J$ such that $\left\{\phi_{i}(x): i \in \Lambda\right\}$ is finite for every $x \in J$. Let $M_{i}$ denote the kernel of $\phi_{i}$. Then we shall suppose that the above representation for $J$ is given so that $\left\{M_{i}: i \in \Lambda\right\}$ is identical with the set of maximal ideals of $J$.

Let $J^{*}$ denote the ring obtained by adjoining $Z_{p}$ to $J$ in the usual manner. Since $J$ has characteristic $p$ then for each $x \in J$, the subring generated by $\boldsymbol{x}$ is isomorphic to a direct sum of a finite number of finite extensions of $Z_{p}$. Thus, there is a nonnegative integer $k(x)$ with $x^{p^{k(x)}}=x$ and hence $J^{*}$ is also a periodic Jordan ring. Without loss of generality we can suppose that $J$ has identity. Further, let $\widetilde{J}$ denote the ring obtained by adjoining to $J$ all elements of the complete direct sum of $\left\{J_{i}: i \in \Lambda\right\}$ in which every projection is either zero or one; that is, $\widetilde{J}$ is formed by adjoining all central idempotents of the complete direct sum to $J$. Now we claim that $\widetilde{J}$ is a periodic Jordan ring and that $\widetilde{J}$ is also isomorphic to a subdirect sum of the rings $J_{i}, i \in \Lambda$. Indeed, if $x$ is in $\widetilde{J}$, then there exist elements $a_{1}, \cdots, a_{n}$ in $J$ and central idempotents $e_{1}, \cdots, e_{n}$ of the complete direct sum such that $x=e_{1} a_{1}+\cdots+e_{n} a_{n}$. Now for each $i \in \Lambda, \phi_{i}\left(a_{j}\right)=\phi_{i}\left(e_{j} a_{j}\right)$ or $\phi_{i}\left(e_{j} a_{j}\right)=0$, and hence if $a_{j}^{n\left(a_{j}\right)}=a_{j}$, then $\left(e_{j} a_{j}\right)^{n\left(a_{j}\right)}=e_{j} a_{j}$. Therefore, for each $i \in \Lambda, \phi_{i}(x)^{n_{i}}=\phi_{i}(x)$ where $n_{i}=$ $n\left(a_{j_{1}}+\cdots+a_{j_{k}}\right)$ and $\left\{a_{j_{1}}, \cdots, a_{j_{k}}\right\}$ is a subset of $\left\{a_{1}, \cdots, a_{n}\right\}$. Hence the set $\left\{n_{i}: i \in \Lambda\right\}$ is finite and $\mathcal{T}$ is periodic. The second assertion follows immediately 
from the fact that $J \subseteq \mathcal{J}$ and that $\mathcal{J}$ is contained in the complete direct sum of the rings $\left\{J_{i}: i \in \Lambda\right\}$.

Our next task is to represent $\widetilde{J}$ as the ring of continuous sections of a sheaf of Jordan rings. Analogous to [3], by a sheaf of nonassociative rings we shall mean a topological space $X$ and a disjoint union $R$ of nonassociative rings with identity $\left\{R_{x}: x \in X\right\}$ with a function $\pi: R \rightarrow X$ given by $\pi\left(r_{x}\right)=x$ if $r_{x} \in R_{x}$. Moreover, $R$ has a topology such that

(i) $\pi$ is a local homeomorphism.

(ii) The function $r \rightarrow-r$ is continuous and the functions $(r, s) \rightarrow$ $r+s,(r, s) \rightarrow r s$ defined on $\left\{(r, s): r, s \in R_{x}\right.$ for some $\left.x \in X\right\}$ are continuous.

(iii) The function $x \rightarrow 1_{x}$, the identity of $R_{x}$, is continuous.

Further, let $\Gamma(X, R)$ denote the set of all continuous functions from $X$ to $R$ such that if $\sigma \in \Gamma(X, R)$, then for each $x \in X, \pi(\sigma(x))=x$. Now for $\sigma, \tau \in \Gamma(X, R)$ define $(\sigma+\tau)(x)=\sigma(x)+\tau(x)$ and $\sigma \tau(x)=\sigma(x) \tau(x)$; then as in [3] $\Gamma(X, R)$ is a nonassociative ring with identity called the ring of continuous sections of the sheaf $R$. Moreover, it is clear that $\Gamma(X, R)$ is a Jordan ring if each $R_{x}$ is a Jordan ring.

We next consider a particular construction of a sheaf of nonassociative rings similar to the one employed in [3]. Let $R$ be a nonassociative ring with identity and let $B(R)$ be the set of central idempotents of $R$. Then $B(R)$ is a Boolean algebra. If $X(R)$ denotes the set of all maximal ideals of $B(R)$, then we can consider $X(R)$ a topological space where the closure $\bar{A}$ of $A$ is $\{M \in X(R)$ : $M \supseteq \bigcap A$ \}. In [4] Stone has proved that $X(R)$ is totally disconnected, compact, Hausdorff space or equivalently, a Boolean space. The sets

$$
N(e)=\{M \in X(R): e \in B(R), e \notin M\}
$$

are exactly the open and closed sets of $X(R)$ and form a basis for the topology. Moreover,

$$
N\left(e_{1}\right) \cap N\left(e_{2}\right)=N\left(e_{1} e_{2}\right), \quad N(1-e)=X(R)-N(e),
$$

for $e, e_{1}, e_{2} \in B(R)$. Finally if $\left\{O_{i}: i \in \Phi\right\}$ is an open covering of $X(R)$, then there exist orthogonal elements $e_{1}, \cdots, e_{n}$ in $B(R)$ such that $N\left(e_{1}\right), \cdots, N\left(e_{n}\right)$ is a disjoint open covering of $X(R)$ and for each $j=1,2, \cdots, n$ there exists $i_{j} \in \Phi$, with $N\left(e_{j}\right) \subseteq O_{i_{j}}$

For each $M \in X(R)$, let $R_{M}=R / R M$ and $R(R)$ be the disjoint union of the rings $R_{M}, M \in X(R)$. Further, for each $a$ in $R$, define $\sigma_{a}: X(R) \rightarrow$ $R(R)$ by $\sigma_{a}(M)$ to be the image of $a$ in $R_{M}$. Now we topologize $R(R)$ with 
the weakest topology such that the sets $\sigma_{a}(N(e))$ are open for all $e \in B(R)$ and $a \in R$. Next we define $\pi: R(R) \rightarrow X(R)$ by $\pi^{-1}(M)=R_{M}$. Then exactly as in [3], $R(R)$ is a sheaf of nonassociative rings. Moreover, $\left\{\sigma_{a}: a \in R\right\}$

$\subseteq \Gamma(X(R), R(R))$. Consider the map $\xi: R \rightarrow \Gamma(X(R), R(R))$ given by $\xi(a)=\sigma_{a}$ for each $a \in R$. It is clear that $\xi$ is a homomorphism of $R$ and the kernel of $\xi$ is just $\bigcap R M$ over all $M \in X(R)$.

LEMMA 2. With the above notation, the kernel of $\xi$ is zero.

Proof. Let $A$ be an abelian group, then we call $A$ an $R$-module if there exists a function mapping $R \times A$ to $A ;(r, a) \rightarrow r a$ for every $r \in R$ and $a \in A$ such that for $r_{1}, r_{2} \in R, a_{1} a_{2} \in A$

$$
\begin{aligned}
r_{1}\left(a_{1}+a_{2}\right) & =r_{1} a_{1}+r_{1} a_{2}, \\
\left(r_{1}+r_{2}\right) a_{1} & =r_{1} a_{1}+r_{2} a_{1}, \\
1 a_{1} & =a_{1} .
\end{aligned}
$$

Moreover, if $r_{1}$ or $r_{2}$ lies in the center of $R$, then $\left(r_{1} r_{2}\right) a_{1}=r_{1}\left(r_{2} a_{1}\right)$. Now with this definition for an $R$-module, Lemmas 1.1 to 1.7 of [3] hold for $R$ and the proofs are exactly the same. Therefore, by Lemma 1.7 of [4],

$$
\bigcap_{M \in X(R)} R M=(0)
$$

and $\xi$ is a monomorphism.

As in [3], since $X(R)$ is a Boolean space, then $\xi$ is in fact an isomorphism of $R$ onto $\Gamma(X(R), R(R))$. As is further proved in [3], $\Gamma(X(R), R(R))$ is a subdirect sum of the rings $\left\{R_{M}: M \in X(R)\right\}$. Finally before returning to the case at hand, we shall need one more fact about $\Gamma(X(R), R(R))$. Let $e_{M}$ be an idempotent in $R_{M}$. Then there exists $a \in R$ with $\sigma_{a}(M)=e_{M}$. Now the set $O=\left\{N \in X(R): \sigma_{a^{2}-a}(N)=0\right\}$ is a neighborhood of $M$ in $X(R)$. Hence there exists $f \in B(R)$ with $M \in N(f) \subseteq O$. Define $\sigma: X(R) \rightarrow R(R)$ by $\sigma(N)=\sigma_{a}(N)$ for all $N \in N(f)$ and $\sigma(N)=0_{N}$ for $N \notin N(f)$. Since $N(f)$ is open and closed, then $\sigma \in \Gamma(X(R), R(R))$ and hence there is an element $e$ of $R$ with $\sigma_{e}=\sigma$. Moreover, $e^{2}=e$ and the image of $e$ in $R_{M}$ is $e_{M}$. Thus, we have proved the following lemma.

LEMMA 3. With the above notation, if $e_{M}$ is an idempotent in $R_{M}$, then there exists an idempotent $e$ of $R$ such that the image of $e$ in $R_{M}$ is $e_{M}$. 
We now return to the Jordan periodic rings $J$ and $\widetilde{J}$ and consider the sheaf of Jordan rings $R(\widetilde{J})$ and ring of continuous sections $\Gamma(X(\widetilde{J}), R(\widetilde{J}))$ which is isomorphic to $\widetilde{J}$. We begin by looking at those rings $\widetilde{J}_{M}$ which are not associative.

LEMMA 4. If $\widetilde{J}_{M}$ is not associative, then $\widetilde{J}_{M}$ is a simple periodic Jordan ring of capacity 2. Moreover $J \cap \widetilde{J} M$ is a maximal ideal of $J$, or $J \cap \widetilde{J} M=J$.

Proof. Since $\widetilde{J}_{M}$ is not associative, then by [5, Corollary 1], $\widetilde{J}_{M}$ has an idempotent $f_{M}$ which is not central. Take $a_{M}$ to be a nonzero element of the 1/2-space of $\widetilde{J}_{M}$ with respect to the Pierce decomposition of $\widetilde{J}_{M}$ given by $f_{M}$. Next we take idempotents $u_{M}$ and $v_{M}$ in $\widetilde{J}_{M}$, such that $u_{M} f_{M}=u_{M}$, $v_{M} f_{M}=0_{M}$, and $a_{M}^{n\left(a_{M}\right)-1}=u_{M}+v_{M}$. Hence $u_{M}$ and $v_{M}$ are orthogonal connected idempotents in $\widetilde{J}_{M}$.

We claim that $u_{M}+v_{M}=1_{M}$, the identity of $\widetilde{J}_{M}$. Let $\phi_{M}$ denote the mapping of $\widetilde{J}$ onto $\widetilde{J}_{M}$. Then if we take $e \in B(\widetilde{J})$ such that $M \in N(e)$ and $u$ idempotent in $\widetilde{J}$ such that $\phi_{M}(u)=u_{M}$ and $u e=u$, then $v=e-u$ is an idempotent in $\widetilde{J}$ orthogonal to $u$ such that $\phi_{M}(v)=1_{M}-u_{M}$. Next choose $b \in \widetilde{J}$ so that $\phi_{M}(b)=a_{M}$ and let $a=2 U_{u, v}(b)$. Then $a \in \widetilde{J}_{1 / 2}(u)$ $\cap \widetilde{J}_{1 / 2}(v)$ and

$$
\phi_{M}(a)=2 U_{u_{M}, 1_{M}-u_{M}}\left(a_{M}\right)=a_{M}
$$

Now $a^{n(a)-1}=g+h$ where $g$ is a nonzero idempotent in $\widetilde{J}_{1}(u)$ and $h$ is a nonzero idempotent in $\widetilde{J}_{1}(v)$, and hence $g$ and $h$ are connected idempotents in $\widetilde{J}$. Thus, by [1], $\widetilde{J}_{1}(g+h)$ is a direct summand of $\widetilde{J}$ and $g+h \in B(\widetilde{J})$. Therefore, $\phi_{M}(g+h)$ is a central idempotent in $\widetilde{J}_{M}$, and furthermore, $\phi_{M}(g) u_{M}$ $=\phi_{M}(g u)=\phi_{M}(g), \phi_{M}(h) u_{M}=0$, and

$$
a_{M}^{n(a)-1}=\phi_{M}(g)+\phi_{M}(h)=u_{M}+v_{M} .
$$

Now the Pierce decomposition of $\widetilde{J}_{M}$ gives a representation of $\widetilde{J}_{M}$ as a vector space direct sum and $u_{M}, \phi_{M}(g)$ lie in the same summand and $v_{M}, \phi_{M}(h)$ lie in the same summand. Hence $u_{M}=\phi_{M}(g)$ and $v_{M}=\phi_{M}(h)$. Now if $u_{M}+v_{M}$ is not the identity of $\widetilde{J}_{M}$, there exists a nonzero $e^{\prime} \in B(\widetilde{J})$ so that $e^{\prime} \vee(g+h)$ $=e$ and $e^{\prime} \wedge(g+h)=0$. But then $e^{\prime} \notin M$ whereas $g+h \notin M$ and $e^{\prime}(g+h)=0 \in M$. However, this contradicts the maximality of $M$ and thus $u_{M}+v_{M}$ is the identity on $\widetilde{J}_{M}$.

We can now prove that $\widetilde{J}_{M}$ has capacity 2 . If this is not the case, then not both $u_{M}$ and $v_{M}$ are primitive. Suppose that there exists a nonzero idempotent $u_{M}^{\prime} \neq u_{M}$ with $u_{M}^{\prime} u_{M}=u_{M}^{\prime}$. Now as above we can suppose that there exists a nonzero $v_{M}^{\prime}$, idempotent, $v_{M}^{\prime} v_{M}=v_{M}^{\prime}$ and $u_{M}^{\prime}, v_{M}^{\prime}$ connected. But then as above $u_{M}^{\prime}+v_{M}^{\prime}=1_{M}$. However, this is impossible since $u_{M}-u_{M}^{\prime}$ is a nonzero idempotent orthogonal to $u_{M}^{\prime}+v_{M}^{\prime}$. Therefore, $\widetilde{J}_{M}$ has capacity 2. 
Now the simplicity of $\widetilde{J}_{M}$ follows immediately from the fact that every nonzero element of the $1 / 2$-space of $\widetilde{J}_{M}$ with respect to $u_{M}$ is a unit in $\widetilde{J}_{M}$.

It remains to prove that $J \cap \widetilde{J} M$ is maximal in $J$ or $J \cap \widetilde{J} M=J$. If not, then the image of $J$ in $\widetilde{J}_{M}$ is not simple. Since $\widetilde{J}_{M}$ has capacity 2 , then if $u$ is an idempotent in $\phi_{M}(J)$, there is at most one nonzero idempotent in $\widetilde{J}_{M}$ orthogonal to $u$. Now if $u$ is the unique nonzero idempotent in $\phi_{M}(J)$, then $\phi_{M}(J)$ is a field and it follows that $J \cap \widetilde{J} M$ is maximal. Hence $\phi_{M}(J)$ must contain another nonzero idempotent $v$. If the $1 / 2$-space of $\phi_{M}(J)$ with respect to $u$ is not zero, then, as above, $\phi_{M}(J)$ must have a pair of connected, orthogonal idempotents which add to the identity and hence $\phi_{M}(J)$ must be simple. But again this implies that $J \cap \widetilde{J} M$ is maximal. Hence $u$ is in the center of $\phi_{M}(J)$. Similarly $v$ is in the center of $\phi_{M}(J)$. Now $u$ and $v-u v$ are orthogonal idempotents in $\phi_{M}(J)$ and hence $u+v-u v=1_{M}$ in $\phi_{M}(J) \subseteq \widetilde{J}_{M}$. Therefore $\phi_{M}(J)$ must be the direct sum of two fields. However, we have that $J$ is isomorphic to a subdirect sum of simple rings $\left\{J_{i}: i \in \Lambda\right\}$ and this was done in such a way that the set of kernels of the projections of $J$ onto $J_{i}, i \in \Lambda$, equals the set of maximal ideals of $J$. Thus, there exist $i$ and $j$ in $\Lambda$ with $\phi_{M}(J)$ isomorphic to $J_{i} \oplus J_{j}$. But $J_{i}$ and $J_{j}$ are direct summands of $\widetilde{J}$ so $\phi_{M}(J)$ is a direct summand of $\widetilde{J}_{M}$ which is impossible since $1_{M} \in \phi_{M}(J)$. And this contradiction implies that $J \cap \widetilde{J} M=J$ or $J \cap \widetilde{J} M$ is a maximal ideal of $J$.

Now before proceeding we remark that Lemma 3 allows us to identify each $\phi_{M}(J) \neq 0$ for which $\widetilde{J}_{M}$ is not associative with a $J_{i}$ for some $i \in \Lambda$, and since $J_{i}$ is a direct summand of $\widetilde{J}$, then without the loss of generality $\widetilde{J}_{M}=J_{i}=\phi_{M}(J)$.

We have remarked earlier that any periodic Jordan ring $J$ of prime characteristic $p$ different from 2 is isomorphic to a subdirect sum of periodic Jordan rings $\left\{J_{i}: i \in \Phi\right\}$ and that each $J_{i}$ can be considered as a subring of $\Omega_{2}^{+}$where $\Omega$ is the algebraic closure of $Z_{p}$. In the next result we shall prove that this can be done in such a way that for each $a \in J,\left\{\phi_{i}(a): i \in \Phi\right\}$ is finite, where $\phi_{i}$ denotes the projection of $J$ onto $J_{i}$.

THEOREM 5. Let $J$ be a prime characteristic periodic Jordan ring. Then there exists a set of simple periodic Jordan rings $\left\{J_{i}: i \in \Delta\right\}$ with each $J_{i} \subseteq \Omega_{2}^{+}$ such that $J$ is isomorphic to a subdirect sum of $\left\{J_{i}: i \in \Delta\right\}$ with projections $\left\{\phi_{i}: i \in \Delta\right\}$. Moreover, for each $a \in J,\left\{\phi_{i}(a): i \in \Delta\right\}$ is finite.

Proof. We begin by considering the representation of $J$ as a subdirect sum of the rings $\left\{J_{i}: i \in \Lambda\right\}$ and by considering the ring $\widetilde{J}$. We have that $\xi: \widetilde{J} \rightarrow$ $\Gamma(X(\widetilde{J}), R(\widetilde{J}))$ is an isomorphism of $\widetilde{J}$ onto a subdirect sum of the rings $\left\{\widetilde{J}_{M}\right.$ : $M \in X(\widetilde{J})\}$ with projections $\phi_{M}$ for each $M \in X(\widetilde{J})$. Moreover, for each $M \in$ $X(\widetilde{J}), \widetilde{J}_{M}$ is either a simple, not associative periodic Jordan ring or associative periodic. Furthermore, if $\widetilde{J}_{M}$ is associative, then we know that $\widetilde{J}_{M}$ is a subdirect 
sum of subfields of $\Omega$. Now define $\left\{\widetilde{J}_{i}: i \in \Phi\right\}$ to be the set of all $\widetilde{J}_{M}$, which are not associative, union the set of all summands of each of the $\widetilde{J}_{M}$ which are associative. The reader should note that we have chosen a prior representation for each of the associative $\widetilde{J}_{M}$ in which each of the summands is contained in $\Omega \subseteq \Omega_{2}^{+}$, and that each not associative $\widetilde{J}_{M}$ is considered to be contained in $\Omega_{2}^{+}$. It is immediate that $\widetilde{J}$ is isomorphic to a subdirect sum of $\left\{\widetilde{J}_{i}: i \in \Phi\right\}$. Let the projection of $\widetilde{J}$ onto $\widetilde{J}_{i}$ be $\psi_{i}$. Then $\widetilde{J}_{i}$ is not associative if and only if $\psi_{i}=\phi_{M}$ for some $M \in X(\widetilde{J})$ such that $\widetilde{J}_{M}$ is not associative. Finally let $\Delta=$ $\left\{i \in \Phi: \psi_{i}(J) \neq 0\right\}$, then $J$ is isomorphic to a subdirect sum of the rings $\left\{\psi_{i}(J)\right.$ : $i \in \Delta$; furthermore each $\psi_{i}(J)$ must be a simple, periodic Jordan ring. We shall now show that this is the desired representation for $J$.

Let $a$ be an element of $J$. For each $i \in \Lambda$, define $\Lambda_{i}=\left\{j \in \Lambda: \phi_{j}(a)=\right.$ $\phi_{i}(a)$ ) and take $e_{i} \in \widetilde{J}$ such that $\phi_{k}\left(e_{i}\right)$ is zero if $k \notin \Lambda_{i}$ and one if $k \in \Lambda_{i}$. Next let $\alpha_{i}=a e_{i}, \alpha_{i} \in \widetilde{J}$, and let $U_{\beta}=\left\{M \in X(\widetilde{J}): \sigma_{\beta}(M)=\sigma_{a}(M)\right\}$ where $\beta$ is a finite sum of the $\alpha_{i}$ or $\beta=0$. We claim that the set $\left\{U_{\beta}\right\}$ is an open covering of $X(\widetilde{J})$. Let $M \in X(\widetilde{J})$. If $\widetilde{J}_{M}$ is not associative, then $M \in U_{0}$ or $\phi_{M}(J)=J_{k}$ for some $k \in \Lambda$, and it follows that $\sigma_{a}(M)=\sigma_{\alpha_{k}}(M)$ or equivalently $M \in U_{\alpha_{k}}$. If $\widetilde{J}_{M}$ is associative and $\sigma_{a}(M) \neq 0$, then $\phi_{M}(J)$ is isomorphic to a subdirect sum of fields $\left\{F_{k}: k \in \Psi\right\}$ with projections $\zeta_{k}$. Now the cardinality of $\left\{\zeta_{k} \cdot \phi_{M}(a): k \in \Psi\right\}$ is less than or equal to the cardinality of the splitting field of $X^{n(a)}-X$ over $Z_{p}$ in $\Omega$ which is finite. Moreover, each $F_{k}$ is a simple homomorphic image of $J$ so that each $F_{k}$ can be identified, with a suitable notation change, to a $J_{k}, k \in \Psi \subseteq \Lambda$. Hence there exists $\beta=$ $\alpha_{i_{1}}+\cdots+\alpha_{i_{n}}$ such that

$$
\Psi \subseteq \bigcup_{j=1}^{n} \Lambda_{i_{j}}
$$

Now it is immediate that $\sigma_{a}(M)=\sigma_{\beta}(M)$ and $M \in U_{\beta}$. Thus, $\left\{U_{\beta}\right\}$ is an open covering of $X(\widetilde{J})$, and since $X(\widetilde{J})$ is a Boolean space, then there exist elements $e_{1}, \cdots, e_{m} \in B(\widetilde{J})$ such that $X(\widetilde{J})=\bigcup_{l=1}^{m} N\left(e_{l}\right), N\left(e_{s}\right) \cap N\left(e_{t}\right)=\varnothing$ if $s \neq t$, and each $N\left(e_{l}\right)$ is contained in some $U_{\beta}$.

We are now in a position to prove that $\left\{\psi_{i}(a): i \in \Delta\right\}$ is finite. As before $\left\{\psi_{i}(a): \psi_{i}(J)\right.$ is associative $\}$ has cardinality less than or equal to the cardinality of the splitting field of $X^{n(a)}-X$ over $Z_{p}$ in $\Omega$. Hence this set must be finite, and it remains to show that $\left\{\psi_{i}(a): \psi_{i}(J)\right.$ is not associative $\} \subseteq\left\{\phi_{M}(a): \widetilde{J}_{M}\right.$ is not associative is finite. But if $\widetilde{J}_{M}$ is not associative, then take $e_{l}$ with $M \in N\left(e_{l}\right) \subset U_{\beta_{l}}$, and hence

$$
\phi_{M}(a)=\sigma_{a}(M)=\sigma_{\beta_{l}}(M)=\phi_{M}\left(\beta_{l}\right)=\alpha_{i_{l}}
$$


since $\widetilde{J}_{M}$ is identical with one of the $J_{i_{l}}, i_{l} \in \Lambda$. Therefore, the cardinality of $\left\{\psi_{i}(a): \psi_{i}(J)\right.$ is not associative $\}$ is less than or equal to $m$. This completes the proof of the theorem.

As an immediate consequence of Theorem 5 and Theorem 13.2 of [1] we have the following result.

THEOREM 6. Any Jordan periodic ring is isomorphic to a subdirect sum of simple Jordan periodic rings $J_{i}, i \in \Lambda$, with the property that if $a$ is an element of the ring and $\phi_{i}$ is the projection of the ring onto $J_{i}$, then $\left\{\phi_{i}(a): i \in \Lambda\right\}$ is finite.

4. The Jordan case. With Theorem 5 we are now able to prove that $J$ is imbeddable in a constructible prime charactersitic periodic Jordan ring.

THEOREM 7. Let $J$ be a prime characteristic, periodic Jordan ring. Then there exists a constructible Jordan ring $\bar{J}$ with $J \subseteq \bar{J}$.

Proof. First, as noted above, we can suppose that the characteristic of $J$ is not 2. Now, by Theorem 5 we know that there exist simple Jordan periodic rings $\left\{J_{i}, i \in \Delta\right\}$ such that $J$ is a subdirect sum of these rings and for each $a \in J,\left\{\psi_{i}(a): i \in \Delta\right\}$ is finite, where $\psi_{i}: J \rightarrow J_{i}$ is the $i$ th projection. Let $K$ denote the complete direct sum of the rings $\left\{J_{i}, i \in \Delta\right\}$ and define $\bar{J}$ to be the set of all elements $x$ of $K$ such that $\left\{\psi_{i}(x): i \in \Delta\right\}$ is finite. Then it is immediate that $\bar{J}$ is a periodic Jordan ring and that $J \subseteq \bar{J}$. Hence, it remains to show that $\bar{J}$ is constructible.

For each nonzero central idempotent $e$ of $\bar{J}$, define $\bar{J}_{e}$ to be the set of all $a$ in $\bar{J}$ such that $\psi_{i}(a)=0$ if $\psi_{i}(e)=0$ and $\left\{\psi_{i}(a): \psi_{i}(e) \neq 0\right\}$ has cardinaltiy one. It is immediate that each $\bar{J}_{e}$ is a subring of $\bar{J}$. We claim that each $\bar{J}_{e}$ is a Jordan ring of a symmetric bilinear form. But this follows immediately from the fact that each $J_{i}$ is a Jordan ring of a symmetric bilinear form, and if $\psi_{i}(e) \neq 0$, then $\psi_{i}$ is a monomorphism of $\bar{J}_{e}$. Moreover, if $f$ is a central idempotent of $\bar{J}$ such that $f \leqslant e$, then $a \rightarrow a f$ gives an imbedding of $\bar{J}_{e}$ into $\bar{J}_{f}$, so that the copy of $\bar{J}_{e}$ in $\Omega_{2}^{+}$is contained in the copy of $\bar{J}_{f}$ in $\Omega_{2}^{+}$. Hence if $B(\bar{J})$ is the set of central idempotents of $\bar{J}$, then let $J^{\prime}$ be the periodic Jordan ring of prime characteristic constructed from $B(\bar{J})$ and $\left\{\bar{J}_{e}: e \in B(\bar{J}), e \neq 0\right\}$.

We claim that $\bar{J}$ is isomorphic to $J^{\prime}$. Define $\psi: \bar{J} \rightarrow J^{\prime}$ as follows. For each $c \in \bar{J}$, let $c_{1}, \cdots, c_{n}$ be the distinct images of $c$ with respect to $\psi_{i}$ for all $i \in \Delta$, and let $e_{j} \in B(\bar{J})$ be given by $\psi_{i}\left(e_{j}\right)=0$ if $\psi_{i}(c) \neq c_{j}$ and $\psi_{i}\left(e_{j}\right)=1$ if $\psi_{i}(c)=c_{j}$. Then $c=c_{1} e_{1}+\cdots+c_{n} e_{n}$ and $c_{j} \in \bar{J}_{e_{j}}$ for each $j=1,2, \cdots, n$. Now let $\psi$ be given by $\psi(c)=c_{1} * e_{1}+\cdots+c_{n} * e_{n}$. By direct computation, $\psi$ is an isomorphism of $\bar{J}$ onto $J^{\prime}$. 
We end this paper with an example. Let $-\mu$ be a nonsquare in $Z_{p}$, $p \neq 2$, and let

$$
K=\left\{\left(\begin{array}{cc}
\alpha & \beta \mu \\
\beta & \gamma
\end{array}\right): \alpha, \beta, \gamma \in Z_{p}\right\}
$$

Then $K$ is a simple periodic Jordan ring. Let $J$ be the subring of the complete direct sum $\widetilde{J}$ of countable copies of $K$ generated by the discrete direct sum of countable copies of $K$ and the element $\xi \in \widetilde{J}$ with

$$
\phi_{i}(\zeta)=\left(\begin{array}{ll}
1 & 0 \\
0 & 0
\end{array}\right)
$$

for all $i \in Z$. It is immediate that $J$ is a periodic Jordan ring of characteristic $p$. We claim that $J$ is not constructible. Indeed, if this were the case then $J$ would contain a central idempotent $e$ such that $\xi e=\xi$. But $\xi$ is contained in no proper ideal of $J$ and hence $e$ is contained in no proper ideal of $J$. However, this implies that $e$ is the identity of $J$. But clearly $J$ has no identity, and indeed $J$ is not constructible.

\section{REFERENCES}

1. J. M. Osborn, Varieties of algebras, Advances in Math. 8 (1972), 163-369. MR 44 \#6775.

2. R. L. Harris, Doctoral Dissertation, University of Iowa, Iowa City, Iowa.

3. R. S. Pierce, Modules over commutative regular rings, Mem. Amer. Math. Soc. No. 70 (1967). MR 36 \#151.

4. M. H. Stone, Applications of the theory of Boolean rings to general topology, Trans. Amer. Math. Soc. 41 (1937), 375-481.

5. J. A. Loustau, Radical extensions of Jordan rings, J. Algebra (to appear).

DEPARTMENT OF MATHEMATICS, HUNTER COLLEGE (CUNY), NEW YORK, NEW YORK 10021 